\title{
Caracterización de la capacidad intelectual, factores sociodemográficos y académicos de estudiantes con alto y bajo desempeño en los exámenes Saber Pro - año 2012
}

\author{
Characterization of the Intellectual Ability, Sociodemographic and Academic Factors \\ of Students with High and Low Performances in the Saber Pro Exam - 2012 \\ Caracterização da capacidade intelectual, fatores sócio-demográficos e acadêmicos \\ de estudantes com alto e baixo desempenho nos exame Saber Pro - ano 2012
}

\author{
Marly Johana Bahamón M., Lizeth Reyes Ruiz* \\ Universidad Simón Bolivar
}

Doi: dx.doi.org/10.12804/apl32.03.2014.01

\section{Resumen}

El presente artículo se deriva del estudio que tuvo por objetivo caracterizar la capacidad intelectual, factores socio-demográficos y académicos de estudiantes con alto y bajo desempeño en los exámenes Saber Pro. Para ello, se analizaron los puntajes reportados por el Instituto Colombiano para el Fomento de la Educación Superior (ICFES) del periodo I y II de 2012 y los datos reportados por el programa de psicología de una Universidad acerca de la capacidad intelectual, características sociodemográficas y académicas de 68 estudiantes que cursaban entre noveno y décimo semestre del pregrado, cuyas edades oscilaron entre los 21 y 52 años, y que presentaron las pruebas en el periodo mencionado. El procesamiento de los datos se realizó mediante el software estadístico SPSS 18.0 aplicando estadígrafos descriptivos y prueba T Student. Los resultados indican que los estudiantes con mejor desempeño tienen unas características sociodemográficas específicas, capacidad intelectual alta, buen desempeño académico, intereses profesionales relacionados con las áreas de la carrera y adecuados hábitos y técnicas de estudio.

Palabras clave: Saber Pro; Icfes; calidad de la educación; factores académicos.

\section{fibstract}

This paper is derived from a study aimed at characterizing the intellectual capacity, socio-demographic and academic factors of students with high and low test performance in the Saber Pro exams. We analyzed the scores reported by the Colombian Institute for the Promotion of Higher Education (ICFES ) for the period I and II of 2012 and the data reported by the psychology program of a University about intellectual ability, sociodemographic and academic characteristics of 68 students who were studying psychology in the ninth and tenth semester, whose ages ranged between 21 and 52 years, and presented the exam in the period mentioned. The da-

\footnotetext{
* Marly Johana Bahamón M., Grupo de investigación Calidad Académica, Universidad Simón Bolívar; Lizeth Reyes Ruiz, Grupo de investigación Calidad Académica, Universidad Simón Bolívar.

La correspondencia relacionada con este artículo debe ser enviada a Marly Johana Bahamón, Calle 59 \# 59-81, Programa de Psicología. Correo electrónico: mbahamon@unisimonbolivar.edu.co, lireyes@unisimonbolivar.edu.co.
}

Para citar este artículo: Bahamón, M. M., \& Reyes, R. L. (2014). Caracterización de la capacidad intelectual, factores sociodemográficos y académicos de estudiantes con alto y bajo desempeño en los exámenes Saber Pro - año 2012. Avances en Psicología Latinoamericana, 32(3), 459-476. doi: dx.doi.org/10.12804/apl32.03.2014.01 
ta processing was performed using SPSS 18.0 statistical software using descriptive statistics and Student $t$ test. The results indicate that students with better performance have specific sociodemographic characteristics, high intellectual ability, good academic performance, career interests related to study areas and appropriate study habits and techniques.

Keywords: Saber Pro; Icfes; quality of education; academic factors.

\section{Resumo}

As políticas educativas sobre a qualidade da educação superior na Colômbia demandam às instituições que seus estudantes próximos a se formar apresentem uma prova nacional denominada Saber Pro para avaliar as competências desenvolvidas durante o processo educativo. Este exame tem impacto na leitura da sociedade sobre o tipo de estudante que se forma como profissional e os processos institucionais de melhora contínua. Neste marco, o desempenho acadêmico é equiparável com os resultados obtidos na prova mencionada, por isso apresenta-se a preocupação por indagar sobre os fatores diferenciadores em estudantes que conseguem um bom desempenho em comparação com aqueles que não. O presente estudo teve por objetivo caracterizar a capacidade intelectual, fatores sócio-demográficos e acadêmicos de estudantes com alto e baixo desempenho nos exames Saber Pro. Para isto analisaram-se as pontuações reportadas pelo Instituto Colombiano para o Fomento da Educação Superior (ICFES) di período I e II do ano 2012 e os dados reportados pelo programa de psicologia da Universidade objeto de estudo sobre capacidade intelectual, características sócio-demográficas e acadêmicas de 68 estudantes que apresentaram as provas no período mencionado. O processamento dos dados realizou-se mediante o software estadístico SPSS 18.0 aplicando estadísticos descritivos e prova T Student. Os resultados indicam que os estudantes com melhor desempenho têm umas características sócio-demográficas específicas, capacidade intelectual alta, bom desempenho acadêmico, interesses profissionais relacionados com as áreas do programa de estudo e adequados hábitos e técnicas de estudo.

Palavras-chave: Saber Pro; Icfes; qualidade da educação; fatores acadêmicos.

\section{Calidad de la educación superior}

La educación es considerada como uno de los procesos más importantes en el desarrollo de los países y por ende es una preocupación esencial para los gobiernos, teniendo en cuenta que de ella depende, en gran medida, su integración al mundo globalizado. Esto se refleja en la necesidad de crear relaciones funcionales entre la academia, la empresa y el estado, cuestión que demanda mayor calidad a las instituciones de educación superior para responder a la exigencia de ciudadanos profesionales mejor formados para afrontar los retos de la actualidad.

En este sentido, el concepto de calidad remite a una triada referida como la preocupación central de la educación dentro de los sistemas educativos en América Latina: mejorar cobertura, calidad y equidad. Es decir, la reorganización de la gestión y el rol de los actores del sistema; el análisis y toma de decisiones sobre los problemas que afectan la calidad de sus procesos y de sus resultados, y el análisis y formulación de las políticas educativas que la posibilitan (Díaz, Valencia, Muñoz, Vivas \& Urrea, 2006).

Así, se han generado diferentes iniciativas para mejorar los procesos mencionados, no obstante, uno de los más debatidos y cuestionados por su naturaleza es el de calidad y, en este marco, la evaluación de la calidad, donde se ha optado por generar diferentes mecanismos de control que van desde la obtención de registros calificados para el funcionamiento de los programas académicos, hasta la evaluación externa de las competencias desarrolladas por los estudiantes.

El Examen Saber Pro (antes llamado ECAES) es quizá la expresión más palpable frente al tema de la evaluación, puesto que rescata la concepción de la educación como un proceso que no incluye de manera exclusiva la adquisición de conocimientos y se concentra en la evaluación de las competencias del futuro profesional y la evaluación de la calidad de las instituciones de educación superior. Bajo estos preceptos, el proyecto Assessment of Higher Education Learning Outcomes, AHELO, coordinado por la Organización para la Cooperación y el Desarrollo Económicos, OCDE, que investiga la viabilidad de Evaluar Resultados de Aprendizaje en 
la Educación Superior, en el que participa Colombia y que impulsa el mejoramiento de los procesos de educación, propone cuatro grupos de competencias principales que sientan la base de un sistema de aprendizaje de excelencia: (a) competencias en disciplinas específicas, (b) competencias genéricas, (c) aprendizaje en contexto y (d) cadena de valor agregado (OCDE \& Banco Mundial, 2012).

Estas competencias le sirven al ICFES (delegada como única autoridad para evaluar a los estudiantes en todos los niveles del sistema educativo colombiano) como referente en la elaboración de las pruebas Saber Pro. Estas pruebas evalúan las competencias básicas y específicas de los estudiantes, cuando están próximos a graduarse de un programa de pregrado en coherencia con el área de conocimiento. Además de lo anterior, el sistema de evaluación Saber Pro tiene por objetivo medir las variables relacionadas con los procesos institucionales y los resultados de los programas educativos para obtener información que sirva como insumo en las decisiones políticas y estrategias educativas (OCDE \& Banco Mundial, 2012). No obstante, este tipo de prueba, por su naturaleza, ha suscitado un amplio debate, en tanto algunos refieren que está más direccionada a la evaluación del conocimiento; la elaboración de ranking de instituciones universitarias derivado de sus resultados y el riesgo de homogenización curricular por constituirse en un referente de evaluación de calidad institucional (Roa \& Valero citados por Viloria 2006).

\section{Desempeño académico y factores asociados}

Si bien, la evaluación externa de las competencias desarrolladas por los estudiantes es importante para la toma de decisiones, existe otro aspecto de interés pedagógico en el panorama educativo: el desempeño o rendimiento académico. Este factor se convierte en el indicador fundamental dentro del proceso de enseñanza aprendizaje para determinar el grado de competencias obtenidas en el curso de una carrera universitaria y por tanto de la calidad de la educación impartida en las instituciones, las cuales serán evaluadas en la educación media (con el examen Saber Once) y en la educación superior (con el examen Saber Pro).
En esta línea, Himmel (citado en Reyes, 2003), equipara el concepto de rendimiento académico con efectividad escolar definiéndolo como el grado de logro de los objetivos establecidos en los programas oficiales de estudio. Por su parte, Hedel (2003) lo define como el nivel de conocimientos demostrados en un área o materia, comparado con la norma de edad o nivel académico. Así, los objetivos propuestos en las instituciones deben guardar coherencia con las políticas educativas vigentes y, por tanto, los niveles de calidad exigidos.

Sobre el tema de desempeño académico en el contexto universitario, la investigación ha intentado relacionar diferentes variables con el fin de entender qué elementos o procesos pueden asociarse a un alto o bajo desempeño para realizar las acciones necesarias que posibiliten su mejoramiento. En este orden de ideas, el recorrido investigativo expone de manera importante la asociación entre rendimiento académico y factores socio-demográficos, individuales, académicos, hasta afectivo motivacionales.

En cuanto a los factores sociodemográficos, algunos autores señalan que ciertas condiciones propician un mejor desempeño. En esta línea, Piñero \& Rodríguez (citados en Hedel, 2003) refieren que la riqueza del contexto del estudiante tiene efectos positivos sobre el rendimiento académico y de manera similar algunos autores indican que el género del estudiante (masculino), su estado civil (soltero), la edad (jóvenes), el tipo de escuela secundaria a la que asistió (privada), el nivel educativo de sus padres (alto), la condición laboral al ingreso, la situación socioeconómica (positiva), estudiar en la misma ciudad de residencia y la situación laboral de su madre son condiciones que pueden intervenir positivamente en el desempeño durante el curso de una carrera universitaria, pero advierten que su influencia no es igual en todos los estudiantes (Chaín citado por Cú \& Aragón, 2006; Esparza, \& López, 2010; Ferreyra, 2007; Reyes, 2005; Reyes, Castañeda \& Pabón, 2012). Este panorama no es claro en Colombia, puesto que los resultados de las investigaciones han sido difusos; ASCOFAPSI por ejemplo, encontró que los mayores puntajes en las pruebas ECAES (actualmente Saber Pro) se relacionan con un mayor estrato socio-económico (Citado en Avendaño, Jiménez \& Senior, 2008). 
Mientras que en otras se refiere que existe una relación negativa entre el Puntaje obtenido en las pruebas Saber Pro (ECAES) y el estrato socioeconómico, lo cual estaría indicando la presencia de altos puntajes en estratos socio-económicos bajos (Cañón, Cortés, López \& Castellanos, 2009; Pereira, Hernández \& Gómez, 2011).

En este sentido, otros estudios reportan que no hay relación significativa entre condiciones sociodemográficas como el estrato, el género, el lugar de residencia, el tipo de escuela a la que se asistió en la secundaria y el rendimiento académico (Birch \& Miller, 2006; Roa \& Vélez, 2005).

Las habilidades cognitivas, de pensamiento o la capacidad intelectual son otro aspecto que se ha tenido en cuenta para esta investigación, por el supuesto de que a mayores habilidades y capacidad de abstracción del estudiante, mayor es el desempeño académico. Dicha afirmación, supone una medida de base para determinar el futuro éxito escolar. Así, la inteligencia ha sido uno de los factores que más se ha correlacionado con el rendimiento académico (Díaz, Morales \& Amador, 2009; Esparza \& López, 2010; Infante, Marín \& Troyano, 2000).

En esta línea, la evidencia empírica ha demostrado que si bien la inteligencia es un factor importante en la comprensión del rendimiento académico, esta no explica más del $50 \%$ del rendimiento humano (Avendaño, Jiménez \& Senior, 2008), pues es bastante frecuente que estudiantes considerados brillantes por su capacidad intelectual no obtengan los mejores resultados escolares, cuestión que ha incitado el re-direccionamiento de la investigación sobre el tema hacia la valoración de factores motivacionales y vocacionales (De la Peza \& García, 2005). Dentro de estos factores, es común tratar de definir el papel de los intereses y preferencias vocacionales, así como las estrategias que el sujeto utiliza para regular su proceso educativo, tales como los hábitos y técnicas utilizadas para estudiar. Por ejemplo, Díaz, Morales \& Amador (2009) en una investigación realizada con estudiantes de psicología señalan una inclinación de estos, por las áreas denominadas como Persuación, Servicio Social y Literario y un menor interés por las áreas Musical, Aire Libre, Mecánico, Cálculo y Científico.

\section{Método}

\section{Diseño}

El presente estudio se ubica en el paradigma empírico analítico con diseño descriptivo comparativo.

\section{Participantes}

La técnica de muestreo fue no probabilística intencional, en esta se definieron los siguientes criterios de inclusión: haber presentado los exámenes Saber Pro en el año 2012 y contar con los resultados de las pruebas aplicadas al ingreso del programa (test de Raven, cuestionario de hábitos y técnicas de estudio e inventario de intereses profesionales). La muestra estuvo conformada por 68 participantes estudiantes del programa de psicología que cursaban entre noveno y décimo semestre del pregrado. Sus edades oscilaron entre los 21 y 52 años con una media de 25.3 y una desviación típica de 5.8, el $60.3 \%$ reportó como lugar de procedencia la misma ciudad en la que cursaban sus estudios, en tanto el $30.9 \%$ provenían de municipios aledaños y el $8.8 \%$ tenían como lugar de residencia municipios de departamentos diferentes y más alejados. Con relación al estrato socioeconómico, la mayoría de los participantes estuvo concentrada en los estratos $2(42.6 \%), 3(32.4 \%)$ y $1(17.6 \%)$ y en menor medida en los estratos 4 (4.4\%) y $6(2.9 \%)$. En cuanto al tipo de colegio en el cual habían cursado sus estudios de educación básica y media el $67.6 \%$ provenían de instituciones educativas del sector público y el $32.4 \%$ de instituciones educativas privadas.

\section{Instrumentos}

Para la ejecución de esta investigación se aplicaron siete instrumentos de los cuales dos (Examen Saber Once y Saber Pro) se constituyen en pruebas externas, es decir, se tomaron los resultados obtenidos por los estudiantes en la aplicación a nivel nacional de estas evaluaciones. Las otras pruebas fueron aplicadas por el personal adscrito al departamento de medición y evaluación de la institución universitaria. 
Examen Saber Once. Es reconocido como el examen de estado de educación media que se aplica con carácter obligatorio desde 1980 a todos los estudiantes de undécimo grado. Está formado por un núcleo común que indaga por medio de 24 preguntas (a excepción de ciencias sociales en las cuales son 30 preguntas) por las áreas de lenguaje, matemáticas, biología, química, física, filosofía, ciencias sociales e inglés, y un componente flexible, en el cual el evaluado selecciona una de las seis opciones disponibles: profundización en lenguaje, matemáticas, biología o ciencias sociales o las pruebas interdisciplinares de medio ambiente o de violencia y sociedad. El componente escogido por el estudiante se evalúa por medio de quince preguntas. En total la prueba consta de 213 ítems (198 de núcleo común y 15 del componente flexible).

Los reportes individuales tienen siete resultados diferentes (Icfes, 2011, p. 13)

1. Puntaje alcanzado en cada una de las pruebas del núcleo común, en una escala de 0 a 100.

2. Puntaje de competencias en cada área del núcleo común, en una escala de 0 a 10, con su correspondiente nivel de desempeño.

3. Puntaje de componentes en cada una de las pruebas del núcleo común, en una escala de 0 a 10 , con su correspondiente nivel de desempeño.

4. Grado de profundización en la prueba respectiva, en una escala de 0 a 10, con su correspondiente nivel de desempeño.

Examen Saber Pro. El Examen de Estado de la Calidad Superior, Saber Pro, es un instrumento de evaluación que aporta a la identificación de múltiples aspectos de la calidad de la educación superior en Colombia. Por tanto, hace parte de los procesos de evaluación que permite identificar al gobierno nacional las fortalezas y debilidades de los servicios educativos que el sector público y privado ofrece a la población en universitaria. Actualmente, el diseño que se ha adoptado para Saber Pro tiene una estructura modular con la cual los programas pueden seleccionar aquellos módulos que evalúen aspectos fundamentales de su formación.
Los módulos de evaluación son instrumentos que valoran competencias consideradas fundamentales para los futuros egresados de programas de formación de educación superior. Algunos módulos evalúan competencias genéricas y otros evalúan competencias específicas, comunes a grupos de programas, ya sea de la misma o de distintas áreas de formación; es decir, campos de problemas que pueden abordar o que comparten distintas formaciones.

Saber Pro utiliza pruebas con preguntas de selección múltiple y respuesta única. Sin embargo, como parte de los módulos de competencias genéricas, se aplica una prueba en la que el estudiante debe redactar un escrito en el cuadernillo que se le entregará el día del examen, de acuerdo con las indicaciones dadas. El examen de calidad de la educación superior Saber Pro, de acuerdo con los nuevos lineamientos propuestos por el Ministerio de Educación Nacional, evalúa a los estudiantes con dos tipos de prueba, una que explora el nivel en competencias genéricas tales como, escritura, razonamiento cuantitativo, lectura crítica, competencias ciudadanas e inglés. Y otra que evalúa competencias específicas comunes a grupos de programa y que se clasifican en grupos de referencia propuestos por el Icfes.

Medida de características sociodemográficas. Se han tomado como características sociodemográficas la edad, el estrato socioeconómico y el tipo de colegio en el cual el estudiante cursó sus estudios de educación media. Estos datos, fueron recuperados del sistema de información de la universidad.

Test de Matrices Progresivas de Raven. Evalúa la capacidad intelectual y se constituye en una serie de pruebas no verbales, que pueden ser administradas en forma individual o grupal y evalúan la habilidad intelectual y de razonamiento basada en materiales de figuras. Miden la habilidad para hacer comparaciones, razonar por analogía y organizar percepciones espaciales dentro de un todo relacionado sistemáticamente. Las Matrices Progresivas de Raven se diseñaron principalmente como una medida del factor " $g$ " de Spearman o inteligencia general, que está constituida por dos componentes identificados como habilidad deductiva y habilidad 
reproductiva (Anastasi \& Urbina, 1998; Raven J. C., Court, J. \& Raven, J. 1996). Los puntos de corte permiten clasificar las puntuaciones en la siguiente escala: Deficiente, Inferior a término medio, término medio, superior al término medio y superior.

Factores académicos. Como factores académicos se delimitaron el desempeño académico, intereses profesionales, hábitos y técnicas de estudio.

Medida de desempeño académico. Se evaluó por medio del informe que ofrece el sistema de rendimiento académico de la universidad, sobre el promedio acumulado que cada estudiante tiene en la carrera que cursa en el momento de la medición. La norma académico-administrativa de la Universidad objeto de estudio establece que dicho promedio debe encontrarse en una escala de 0 a 5 , donde puntuaciones inferiores a 3.0 son consideradas como bajas, las puntuaciones entre 3.0 y 3.9 son medias, mientras que aquellas por encima de 4.0 son consideradas altas.

Inventario de intereses profesionales (IPP) de Thurstone. Fue diseñado para servir de instrumento de orientación en la elección de las actividades para relacionarlas con las orientaciones vocacionales del evaluado. Se trata de un inventario de profesiones que consta de 100 casillas en las que aparecen parejas de profesiones entre las que el sujeto debe elegir. Este instrumento permite reforzar la comprensión sobre la capacidad intelectual de los estudiantes al explorar sus intereses incluyendo aspectos motivacionales asociados. El conjunto de ocupaciones trata de determinar el grado de interés relativo en cada uno de los diez siguientes factores o capos de actividades (Thurstone, 1989):

Tabla 1

Factores del IPP

\begin{tabular}{ll}
\hline Factor & \multicolumn{1}{c}{ Descripción } \\
\hline F & $\begin{array}{l}\text { Ciencia física y química, astronomía, ingeniería, } \\
\text { técnicas afines. }\end{array}$ \\
B & $\begin{array}{l}\text { Ciencias biológicas, medicina, fisiología, } \\
\text { zoología, botánica. }\end{array}$
\end{tabular}

\begin{tabular}{ll}
\hline Factor & \multicolumn{1}{c}{ Descripción } \\
\hline C & Cálculo, estadística, economía. \\
E & $\begin{array}{l}\text { Actividades ejecutivas: dirección, organización, } \\
\text { supervisión. }\end{array}$ \\
P & $\begin{array}{l}\text { Actividades persuasivas: política, jurídica, } \\
\text { comercial. }\end{array}$ \\
L & $\begin{array}{l}\text { Actividades lingüísticas: periodismo, } \\
\text { información, recopilación o exposición de hechos } \\
\text { pasados o presentes. }\end{array}$ \\
H & $\begin{array}{l}\text { Actividades humanitarias: disposición altruista y } \\
\text { elevación de miras en el trato con los demás. }\end{array}$ \\
A & $\begin{array}{l}\text { Artes plásticas: decoración, diseño o dibujo } \\
\text { artístico-comercial. }\end{array}$ \\
M & Música: composición, ejecución o enseñanza.
\end{tabular}

Fuente: basado en Thurstone, 1989.

Cuestionario de hábitos y técnicas de estudio (CHTE). Tiene como finalidad de realizar un diagnóstico de los factores involucrados en la tarea de estudio. El instrumento evalúa tres aspectos fundamentales:

1. Las condiciones físicas y ambientales

2. La planificación y estructuración del tiempo

3. El conocimiento de las técnicas básicas.

Estos aspectos se desglosan en siete escalas: actitud general hacia el estudio, el lugar de estudio, el estado físico del escolar, plan de trabajo, técnicas de estudio, exámenes y ejercicios, trabajos.

\section{Resultados}

\section{Hallazgos sobre capacidad intelectual}

Relacionado con la capacidad intelectual de los estudiantes, en general se encontró que la mayoría de ellos se clasificaron en puntajes correspondientes al término medio (36.8\%) y superior al medio $(30.9 \%)$ mientras que los menores puntajes en que se ubicaron los estudiantes fueron los designados a deficiente $(4.4 \%)$ y superior $(1.5 \%)$. 
El análisis de los datos en función del desempeño en las pruebas Saber Pro encontró que los estudiantes con mejor rendimiento se ubicaron en su mayoría en término superior al medio $(60 \%)$ y medio (30\%), a diferencia de los datos en estudiantes con menor desempeño puesto que su distribución se ubicó en la categoría término medio e inferior al término medio $(31 \%)$.

\section{Hallazgos sobre características socio-demográficas}

Para iniciar, los participantes que presentaron puntajes bajos en el examen Saber Pro tuvieron una distribución de edad entre los 21 y los 47 años en el que la mayor concentración de estudiantes se encontró en los 23 (29.7\%), 24 (18.9), 22 (10.8) y 26 años (10.8\%). En tanto los participantes con puntajes altos se ubicaron en su mayoría en los 24 años de edad (33.3), los 22.23 y 26 años (22.2\%) respectivamente, con un rango general comprendido entre los 22 y los 26 años.

En cuanto a la ciudad de procedencia, los datos sugieren que quienes tuvieron menor desempeño en las pruebas, en su mayoría (49\%), se encontraban radicados en la misma ciudad en la que estudiaban, seguidos de quienes provenían de otros municipios del mismo departamento ( $26 \%$ ) y solo una minoría (25\%) procedían de otros departamentos. Respecto a quienes tuvieron mejor desempeño, se encontró que el $53 \%$ vivían en la misma ciudad en la cual realizaban sus estudios, seguido de quienes provenían de municipios del mismo departamento en el que los participantes estudiaban (46\%).
Sobre el estrato socio-económico, los participantes se concentraron en los estratos uno al cuatro. En quienes obtuvieron puntuaciones bajas, el estrato socioeconómico reportado fue el dos, a diferencia de los participantes que presentaron puntajes altos pues, se ubicaron en el estrato tres.

Con respecto al tipo de colegio en que cursaron sus estudios de educación secundaria, los sujetos con altos puntajes reportaron en su mayoría pertenecer a instituciones de carácter privado, a diferencia de los que tuvieron menor desempeño quienes informaron provenir de instituciones educativas oficiales.

Hallazgos sobre los factores académicos: resultados obtenidos en evaluaciones externas (Saber Once y Saber Pro).

Sobre la medida de desempeño académico, se tomaron como puntos de referencia los siguientes rangos: desempeño bajo (1-2.9); desempeño medio (3.0-3.9) y desempeño alto (4.0-5.0). En este campo los participantes se ubicaron principalmente en desempeño alto (60.3\%) y medio (39.7\%), sin presencia de desempeño bajo, lo cual es coherente con las políticas de la universidad frente a los requisitos para optar al título de pregrado, pues de lo contrario se indicaría que el estudiante no obtuvo los resultados esperados en algunas asignaturas.

Sobre los resultados del examen Saber Once (véase tabla 2) es posible identificar que las menores puntuaciones, y por tanto de menor desempeño, se encontraron en el componente de filosofía, a diferencia de los componentes de biología y química en los cuales los participantes presentaron mejores puntuaciones.

Tabla 2

Resultados Examen Saber Once

\begin{tabular}{|c|c|c|c|c|c|c|c|c|}
\hline & Biología & Matemáticas & Filosofía & Física & Historia & Química & Lenguaje & Geografía \\
\hline & $\%$ & $\%$ & $\%$ & $\%$ & $\%$ & $\%$ & $\%$ & $\%$ \\
\hline Bajo & 14.7 & 13.2 & 16.2 & 14.7 & 7.4 & 14.7 & 14.7 & 11.8 \\
\hline Medio & 64.7 & 72.1 & 64.7 & 66.2 & 80.9 & 64.7 & 76.5 & 73.5 \\
\hline Alto & 19.1 & 13.2 & 17.6 & 17.6 & 10.3 & 19.1 & 7.4 & 13.2 \\
\hline \multirow[t]{2}{*}{ Total } & 98.5 & 98.5 & 98.5 & 98.5 & 98.5 & 98.5 & 98.5 & 98.5 \\
\hline & 100.0 & 100.0 & 100.0 & 100.0 & 100.0 & 100.0 & 100.0 & 100.0 \\
\hline
\end{tabular}

Fuente: Elaborado por la autora con base en los datos. 
Tabla 3

Resultados Examen Saber Pro

\begin{tabular}{|c|c|c|c|c|c|c|c|c|c|c|c|}
\hline & & \multicolumn{2}{|c|}{$\begin{array}{c}\text { Competencias } \\
\text { ciudadanas }\end{array}$} & \multicolumn{2}{|c|}{ Escritura } & \multicolumn{2}{|c|}{ Inglés } & \multicolumn{2}{|c|}{ Lectura crítica } & \multicolumn{2}{|c|}{$\begin{array}{c}\text { Razonamiento } \\
\text { cuantitativo }\end{array}$} \\
\hline & & $F$ & $\%$ & $F$ & $\%$ & $F$ & $\%$ & $F$ & $\%$ & $F$ & $\%$ \\
\hline \multirow{6}{*}{ Válidos } & Muy bajo & 26 & 38.2 & 17 & 25.0 & 26 & 38.2 & 17 & 25.0 & 23 & 33.8 \\
\hline & Bajo & 19 & 27.9 & 12 & 17.6 & 11 & 16.2 & 18 & 26.5 & 19 & 27.9 \\
\hline & Medio & 14 & 20.6 & 16 & 23.5 & 17 & 25.0 & 16 & 23.5 & 17 & 25.0 \\
\hline & Alto & 6 & 8.8 & 11 & 16.2 & 10 & 14.7 & 13 & 19.1 & 7 & 10.3 \\
\hline & Muy alto & 3 & 4.4 & 10 & 14.7 & 4 & 5.9 & 4 & 5.9 & 2 & 2.9 \\
\hline & Total & 68 & 100.0 & 66 & 97.1 & 68 & 100.0 & 68 & 100.0 & 68 & 100.0 \\
\hline Perdidos & Sistema & 0 & 0 & 2 & 2.9 & 0 & 0 & 0 & 0 & 0 & 0 \\
\hline
\end{tabular}

Fuente: Basado en los datos

En cuanto al análisis de los resultados generales en este examen, es decir del promedio, se evidenció que el $57 \%$ de los participantes se ubicaron en puntuaciones bajas, en tanto solo el $26.5 \%$ mostró puntuaciones altas.

Sobre los puntajes en el examen Saber Pro los participantes del estudio presentaron puntuaciones altas en las competencias de escritura y lectura crítica, cuestión que puede relacionarse con la naturaleza de la disciplina y las demandas de lectura y análisis a las que el estudiante debe responder durante el desarrollo de su carrera. Por otro lado, las competencias que evidenciaron menor desempeño por la obtención de bajas puntuaciones fueron competencias ciudadanas, inglés y razonamiento cuantitativo.

\section{Hallazgos sobre los factores académicos: intereses profesionales}

En lo concerniente a intereses profesionales en estudiantes del programa de psicología que hicieron parte de esta investigación se evidencia que las puntuaciones más altas se concentran en las ciencias físico-químicas y las artes plásticas. No obstante, Los puntajes medios evidencian mayor interés por actividades persuasivas y lingüísticas (véase tabla 4).

En cuanto a las características demostradas por los estudiantes de acuerdo con los puntajes obte- nidos en las pruebas Saber Pro, los datos arrojan información interesante, puesto que los sujetos que mostraron puntajes altos en dicho examen evidenciaron mayor preferencia por actividades lingüísticas, humanitarias y artes plásticas. A diferencia de quienes tuvieron un bajo desempeño que mostraron mayor preferencia por actividades relacionadas con ciencias físicas y químicas.

\section{Hallazgos sobre los factores académicos: hábitos y técnicas de estudio}

En general los participantes del estudio se mostraron como estudiantes aceptables $(45.6 \%)$ y buenos estudiantes (23\%), lo cual expone una actitud positiva hacia el estudio, adecuación del lugar de estudio, capacidad para formular planes de trabajo y uso eficiente de técnicas estudio, así solo un $19,1 \%$ evidenció dificultad para estudiar.

En cuanto a los participantes con mejor desempeño en la prueba Saber Pro se encontró que tienen hábitos de estudio adecuados y hacen uso de técnicas de estudio con regularidad (sus puntajes solo se ubicaron en medio y alto $50 \%$ cada uno). En contraste, los estudiantes con menor desempeño en las pruebas mostraron tener dificultad para adecuarse a las condiciones físicas del ambiente, planificar su trabajo y usar estrategias de trabajo para facilitar el proceso de aprendizaje $(66.7 \%)$. 

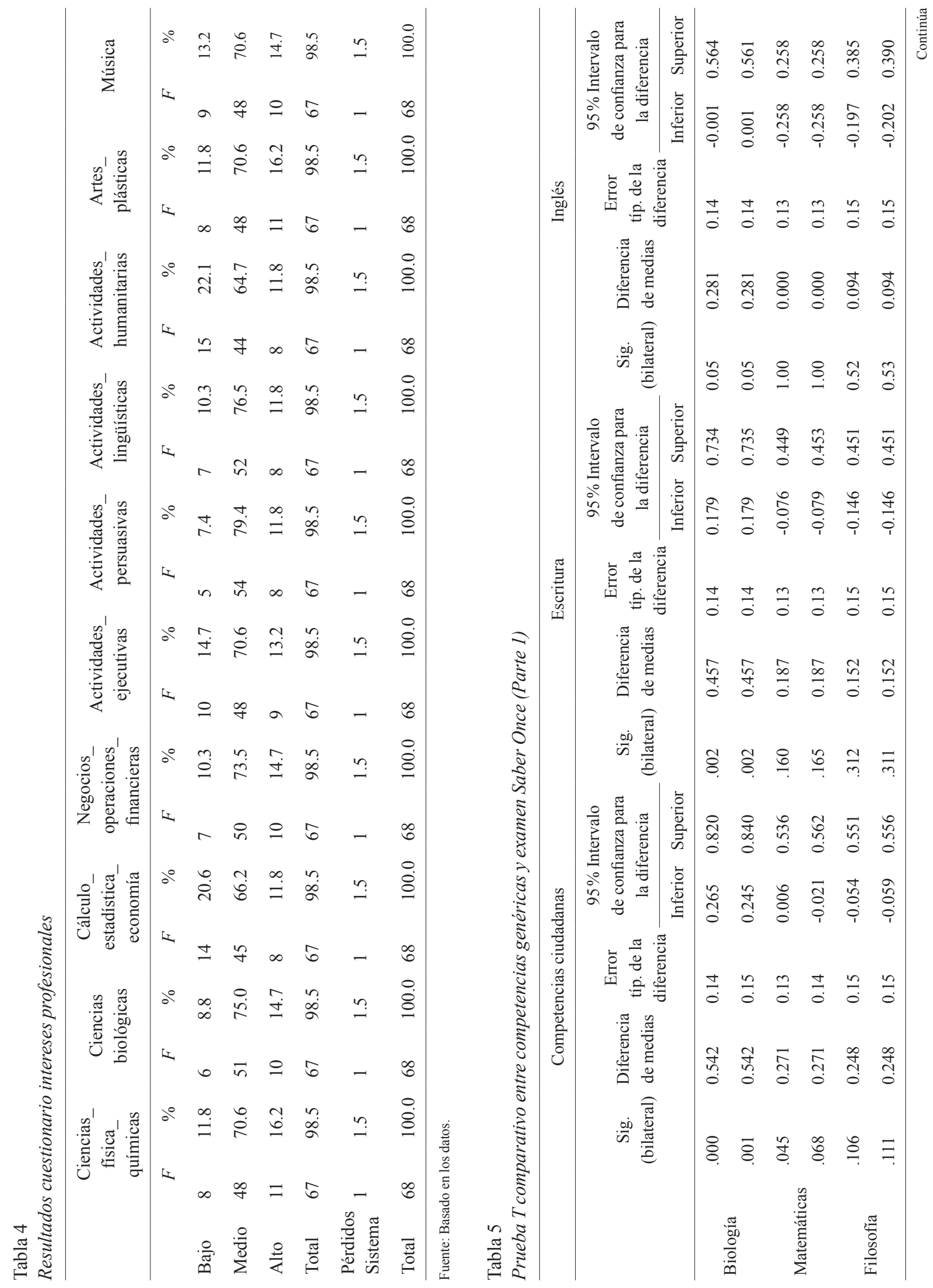


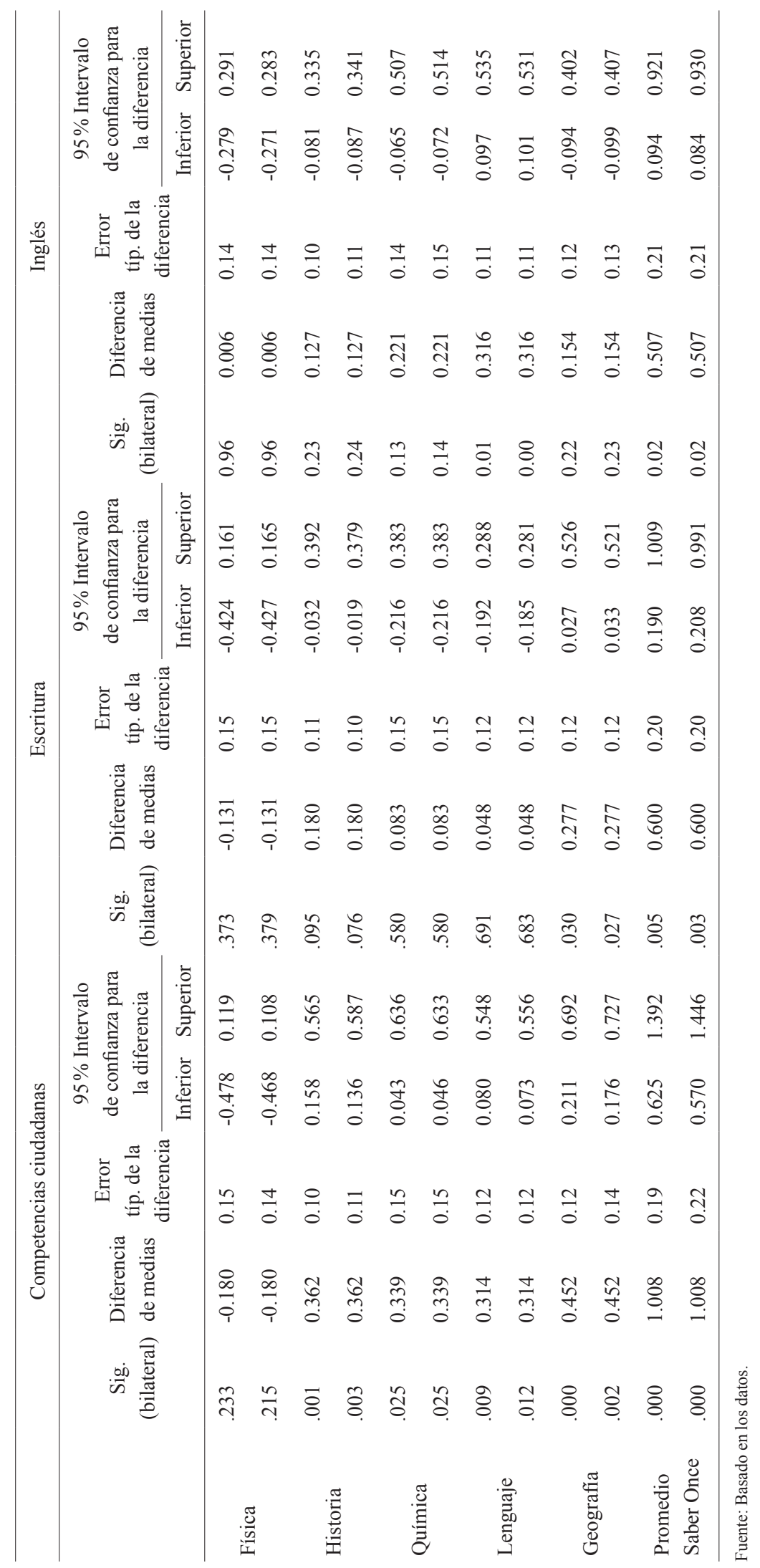




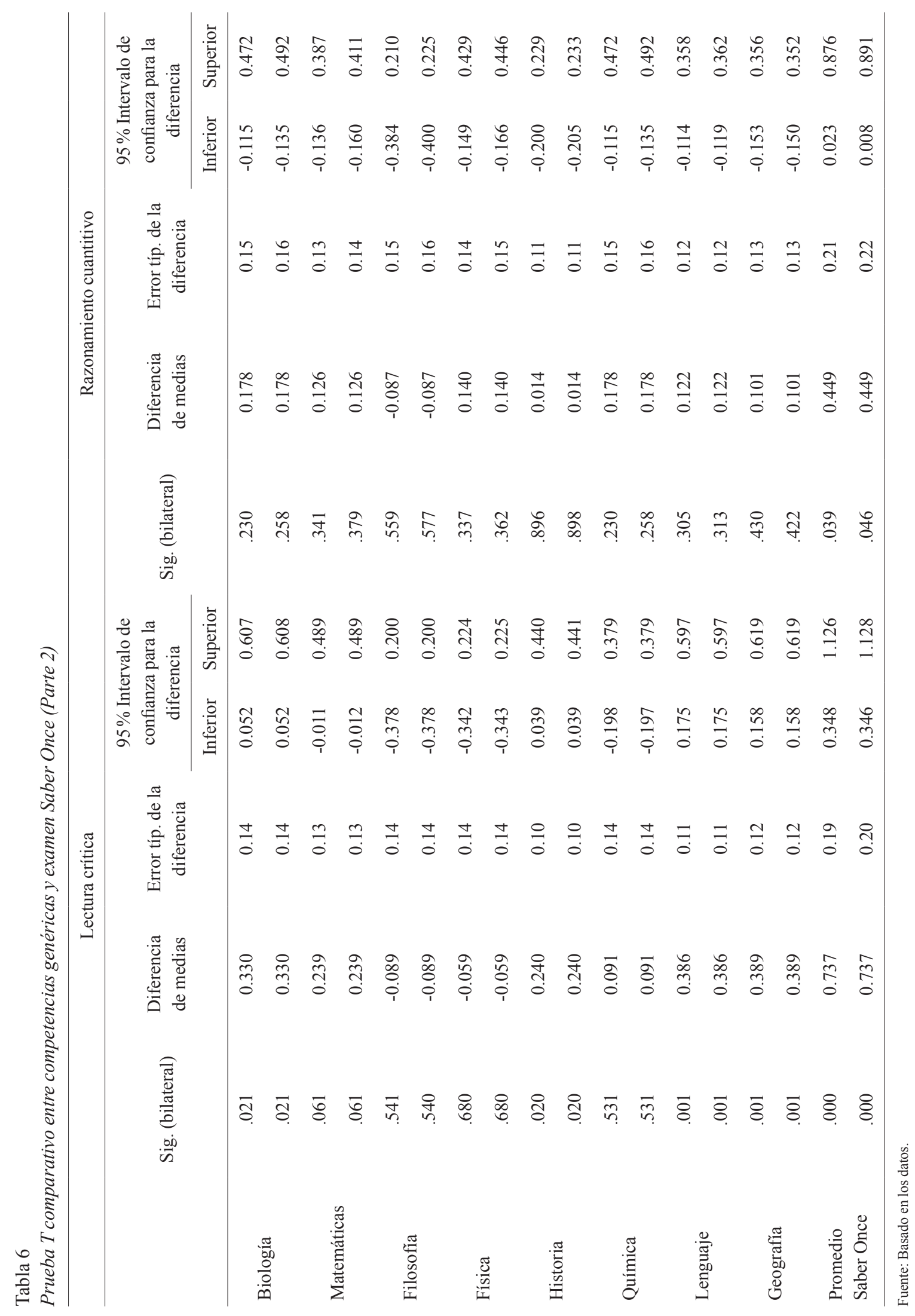


Para realizar los comparativos entre la muestra de estudiantes que presentaron alto y bajo desempeño en las pruebas Saber Pro, en comparación con los resultados de las pruebas Saber Once, se analizaron de manera individual los puntajes de las competencias evaluadas por medio de una Prueba T Student. En los resultados se hallaron diferencias significativas entre los puntajes de competencias ciudadanas y física; escritura y química y lenguaje; inglés y matemáticas, filosofía y física. También se encontraron diferencias significativas ente lectura crítica y física y química; razonamiento cuantitativo y filosofía e historia.

El análisis comparativo entre el desempeño de los participantes en el examen Saber Pro y las características académicas e intelectuales permitió determinar diferencias significativas entre competencias ciudadanas y el interés por actividades de cálculo-estadística, negocios-financieras y ejecutivas; escritura e interés por ciencias biológicas, negocios financieros y artes plásticas; inglés y actividades relacionadas con el cálculo, la lingüística y las actividades humanitarias. Otras diferencias se enfocaron hacia lecturas críticas y ciencias físicas y finalmente los puntajes obtenidos en la competencia de razonamiento cuantitativo y negocios financieros, actividades ejecutivas, actividades persuasivas, artes plásticas. Finalmente, los hábitos y técnicas de estudio mostraron diferencia en los puntajes frente a competencias ciudadanas, inglés y razonamiento cuantitativo.

\section{Discusión}

Sobre las habilidades cognitivas, los resultados indican que la mayoría de los participantes se ubicaron en término medio y superior al medio respectivamente, cuestión que permite inferir la necesidad de una estructura de soporte para la ejecución de actividades cognitivas formales de aprendizaje y, por tanto, de un buen rendimiento o desempeño académico. Estos resultados tienen coherencia con lo expresado por Avendaño, Jiménez \& Senior (2008), quienes definen que no solo la capacidad intelectual del sujeto puede predecir su rendimiento. Así, es posible identificar casos de estudiantes con capacidades intelectuales superio- res a la normalidad estadística y bajo rendimiento académico, o lo contrario, estudiantes con pocas habilidades cognitivas y excelente rendimiento en actividades de carácter académico. Lo anterior pone en evidencia que las instituciones de educación superior pueden evaluar las capacidades de los aspirantes con el objeto de identificar necesidades o requerimientos inmediatos, pero difícilmente para excluir de los procesos educativos a aquellos que presenten menores puntuaciones en las pruebas, pues son diferentes los factores que pueden contribuir a la explicación sobre el desempeño de los estudiantes.

Otros de los aspectos evaluados fueron las características sociodemográficas, campo en el cual existen diferentes posiciones sobre su dinámica frente al desempeño académico. Así, autores como Piñero \& Rodríguez, (citados por Hedel, 2003) y Cañón, Cortés, López \& Castellanos (2009) expresan que las condiciones sociodemográficas y económicas positivas pueden favorecer el desempeño académico, advirtiendo que su influencia no es igual en todos los estudiantes. A diferencia de otros autores (Roa \& Vélez, 2005; Birch \& Miller, 2006) que expresan la inexistencia de relación entre las dos variables y otros que han encontrado relaciones negativas entre el estrato socioeconómico y el desempeño (Pereira, Hernández \& Gómez, 2011). $\mathrm{Al}$ respecto, en esta investigación se encontró que los estudiantes con altas puntuaciones se ubicaron entre los 22 y los 26 años, provenían de municipios cercanos a la ciudad en la cual se realizaban los estudios, la mayoría se ubicaron en estrato socioeconómico tres y la institución educativa en la cual cursaron sus estudios fue de carácter privado. Estos datos deben su explicación en parte a las condiciones y características propias de los estudiantes de la universidad que por su filosofía rescata la importancia de ofrecer servicios a los estratos menos favorecidos de la región en que se encuentra ubicada. Así, los estratos socio-económicos de los cuales provienen la mayoría de los estudiantes son dos y tres. No obstante, es importante referenciar la posibilidad de mejor desempeño ante mejores condiciones para acceder a recursos tecnológicos para la búsqueda de información y mayor estimulación educativa en contextos formales y familiares. De la misma for- 


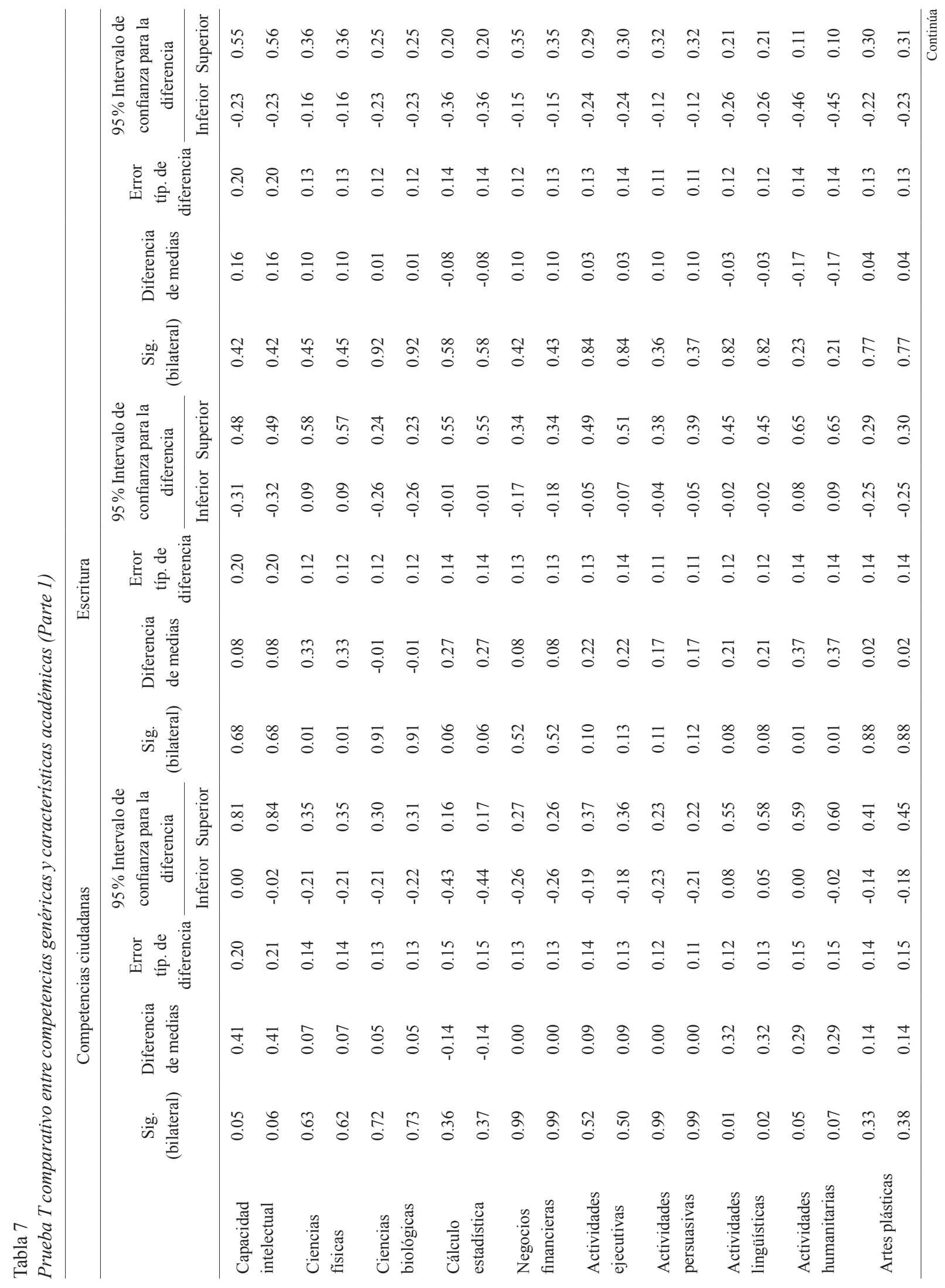



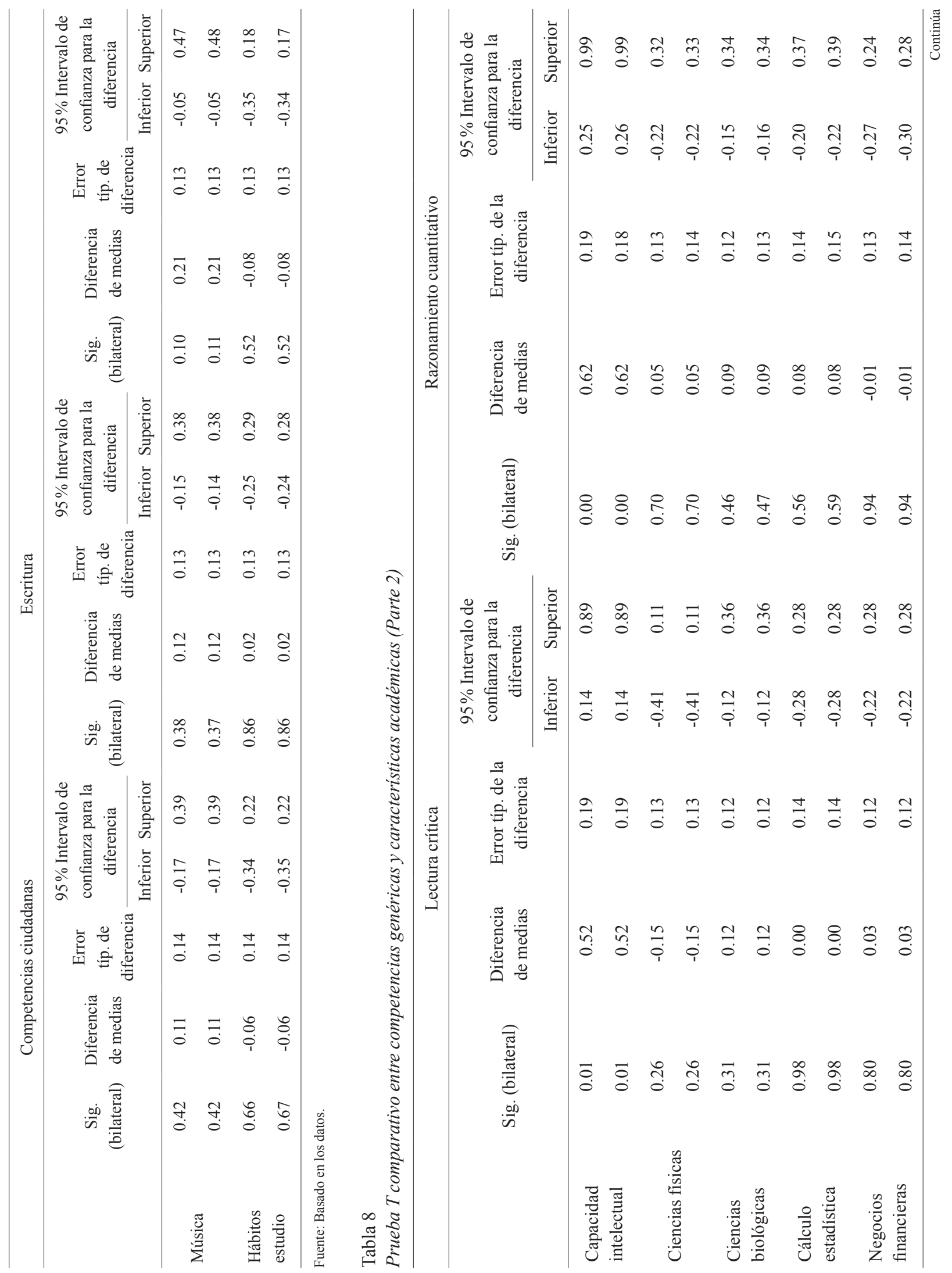
弯

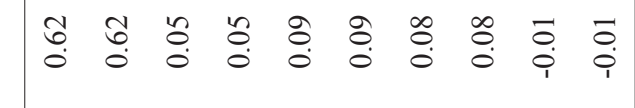

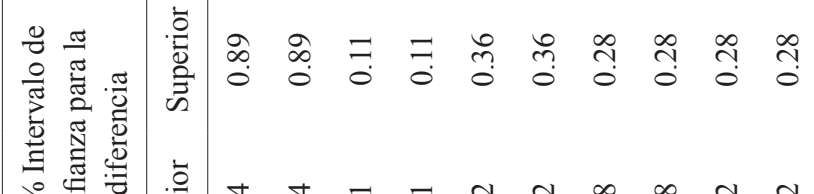
ํํㅇ

莺 $\frac{\pi}{8}$

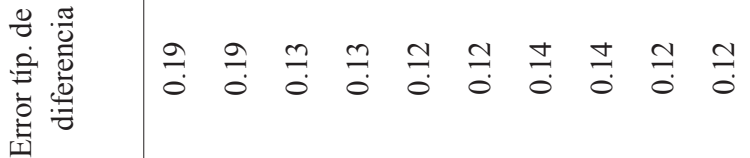
点

일 id

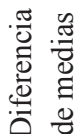

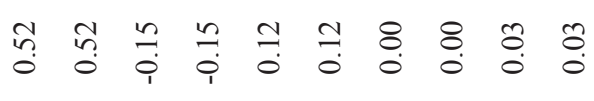

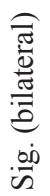

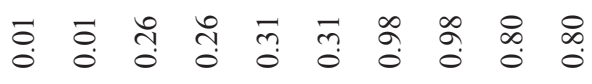

\begin{tabular}{|c|c|c|c|}
\hline 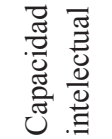 & 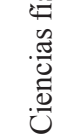 & 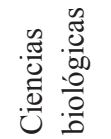 & 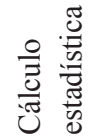 \\
\hline
\end{tabular}




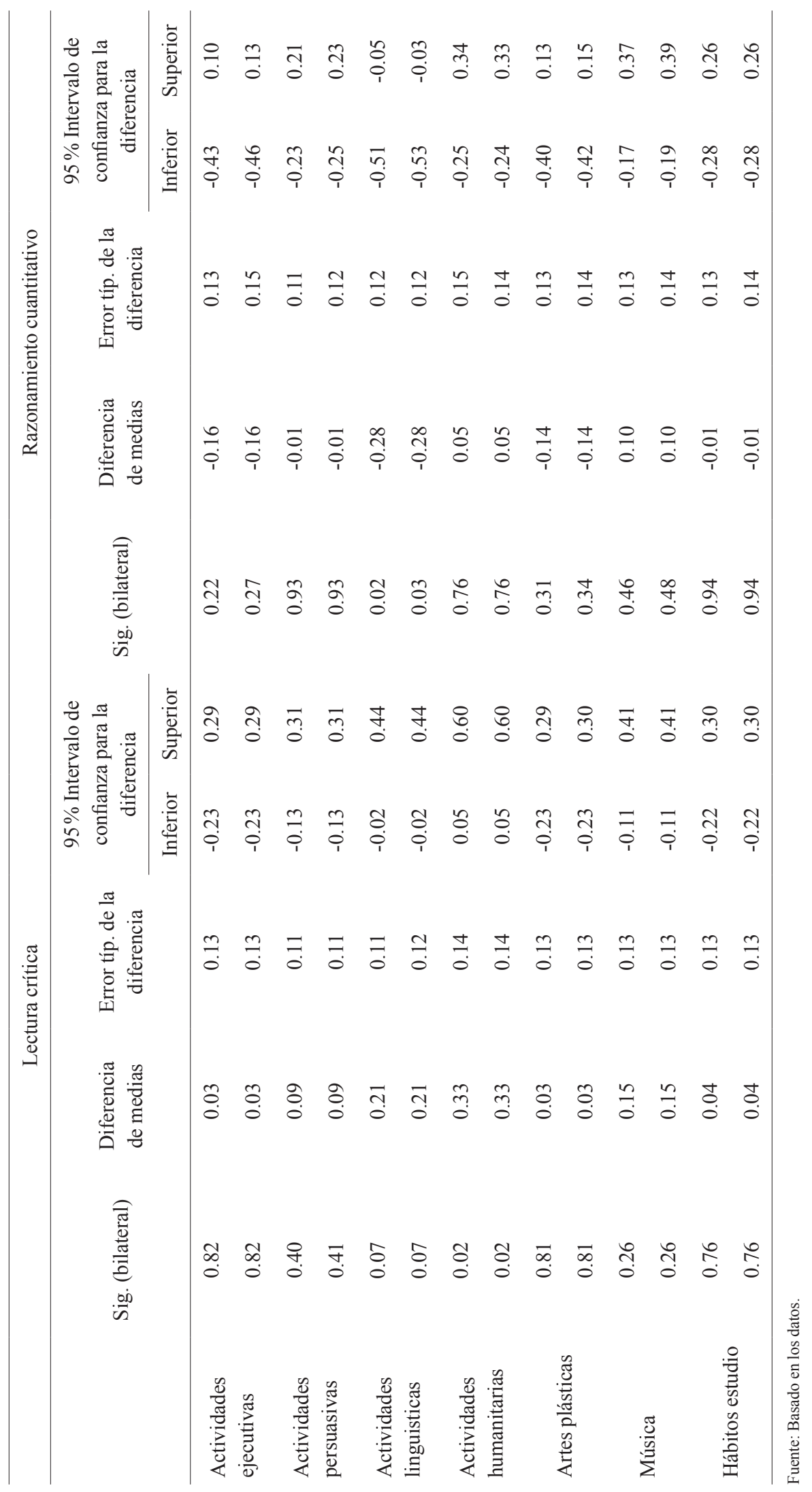


ma, se pone en evidencia que aquellos estudiantes que se encuentran insertos en su contexto de origen o cercanos a este presentan mejor desempeño que quienes tienen que alejarse de su entorno inmediato; esto puede explicarse por la falta de un sistema de apoyo que brinde el soporte necesario a aquellos que deben sufrir el desarraigo social y familiar para poder llevar a cabo sus estudios. Así, este aspecto debe tenerse en cuenta con mayor propiedad al interior de las instituciones de educación superior para generar alternativas que cubran esta necesidad en el estudiantes, información que coincide con lo encontrado por Torres \& Rodríguez (2006) y Garzón, Rojas, Del Riesgo, Pinzón \& Salamanca (2010). En este sentido, emerge la importancia de articular programas desarrollados por departamentos o programas institucionales de bienestar estudiantil que brinden alternativas de apoyo para reducir el riesgo académico de los estudiantes. Otro aspecto a tener en cuenta en futuras investigaciones se relaciona con la calidad de la educación privada frente a las instituciones del sector público, ello debido al buen desempeño mostrado por estudiantes provenientes del sector privado y las marcadas limitantes de los estudiantes que han desarrollado sus estudios secundarios en colegios oficiales. Sobre este aspecto se debe considerar la eficacia de programas para nivelar estas deficiencias al interior de la universidad, pues aun cuando existan se debe evaluar su impacto con relación al desempeño académico de los estudiantes.

En cuanto al desempeño en el examen Saber Once, prueba que deben presentar todos los estudiantes al terminar su proceso académico en el ciclo de bachillerato y que es requerida por las universidades como un requisito obligatorio para su ingreso a cualquier programa de pregrado, se identificaron mayormente puntuaciones bajas, cuestión que permitió identificar el grado de desempeño con el cual los estudiantes iniciaron su proceso universitario, en contraste con los resultados de las pruebas Saber Pro, puesto que los participantes del estudio presentaron puntuaciones altas en algunas competencias (escritura y lectura crítica), cuestión que puede indicar modificaciones en el proceso del estudiante, que le permitieron mejorar su desempeño.
Por lo anterior, se recomienda a los estudiantes asistir a las actividades programadas que buscan mejorar las habilidades, competencias y las técnicas de estudios empleando los siguientes métodos: Talleres de compresión lectora, técnicas de estudios, talleres de profundización y el proceso terapéutico. De igual manera, se recomienda a los docentes, motivar a los estudiantes dentro del aula de clases para que identifiquen los beneficios que estos talleres les brindan.

En cuanto a los intereses profesionales fue posible identificar que las puntuaciones medias y altas se concentraron en actividades persuasivas y lingüísticas, ciencias de físico-químicas y las artes plásticas. Cuestión que guarda relación con los contenidos y exigencias propias de la carrera de psicología, a excepción de ciencias fisicoquímicas. No obstante, aquellos participantes que obtuvieron mayores puntajes en las pruebas Saber Pro, evidenciaron mayor preferencia por actividades lingüísticas, humanitarias y artes plásticas. Resultado que podría indicar que el interés y motivación hacia determinadas áreas del conocimiento puede relacionarse con el desempeño que se obtenga en estas.

Así mismo, aquellos participantes con mejor desempeño en la prueba Saber Pro mostraron que tienen hábitos de estudio adecuados y hacen uso de técnicas de estudio con regularidad, a diferencia de los estudiantes con menor desempeño en las pruebas, quienes mostraron tener dificultad para adecuarse a las condiciones físicas del ambiente, planificar su trabajo y usar estrategias de trabajo para facilitar el proceso de aprendizaje, ello en coherencia a lo encontrado por Hernández y Pozo (1999) y Contreras (2005).

Al realizar el análisis sobre los factores asociados con el alto o bajo rendimiento en las pruebas Saber Pro, es posible identificar que si bien las habilidades cognitivas del estudiante son importantes para obtener un buen desempeño, este es apenas un insumo que contribuye en los procesos de aprendizaje, así, independientemente de la capacidad mental de los estudiantes, es evidente la necesidad de una estructura de soporte para la ejecución de actividades cognitivas formales de aprendizaje, si se 
quiere obtener un buen rendimiento o desempeño académico en los estudiantes.

Las Instituciones de Educación Superior deben diseñar un sistema de apoyo de bienestar estudiantil, articulando programas desarrollados por departamentos o programas institucionales, para reducir el riesgo académico de aquellos estudiantes que deben sufrir el desarraigo social y familiar para poder llevar a cabo sus estudios. $\mathrm{O}$ en su defecto, evaluar el impacto de las estrategias implementadas en las instituciones para tal fin, en aras de determinar el grado de eficacia de estos.

Las Instituciones de Educación Superior están en la obligación de diseñar rutas de apoyo a los estudiantes donde se programen actividades para mejorar las habilidades, competencias y las técnicas de estudios empleando los siguientes métodos: Talleres de compresión lectora, hábitos y técnicas de estudios, talleres de profundización y el proceso terapéutico.

Los docentes, como facilitadores del proceso de formación profesional, están llamados a motivar a los estudiantes dentro del aula de clases para que se concienticen de los beneficios de estos talleres.

\section{Referencias}

Anastasi, A., \& Urbina, S. (1998). Test Psicológicos. Ciudad: Ed. Prentice Hall.

American Psychological Association (1998). Manual de Estilo de publicaciones. México: Ed. Manual Moderno.

Avendaño, B., Jiménez, M., \& Senior, D. (2008). Caracterización de un grupo de estudiante que obtuvo altos puntajes en el examen de calidad de la educación superior ECAES, años 2003-2006. Revista Suma Psicológica, 15(2), 355-384.

Birch, E., \& Miller, P. (2006). Student Outcomes at University in Australia: a Quantile Regression Approach. Australian Economic Papers, 45(1), 1-17.

Cañón, H., Cortés, R., López, M., \& Castellanos, F. (2009). Rendimiento académico y desempeño en el Examen de Calidad de la Educación Superior (ECAES) de enfermería, año 2003. Revista de Enfermería, 11(1), 67-80.
Contreras, F., Espinosa, J., Haikal, A., Esguerra, G., Polanía, A, \& Rodríguez, A. (2005). Autoeficacia, Ansiedad y Rendimiento Académico en adolescentes. Diversitas: Prespectivas en psicología, 1(2), 183-194.

Cú, G., \& Aragón, F. (2006). El perfil sociodemográfico e impacto en el rendimiento académico de los alumnos de la Universidad Autónoma de Campeche, México. Revista Nuevas tecnologías y sociedad, 42, 1-10.

De la Peza, R., \& García, E. (2005). Relación entre variables cognitivo emocionales y rendimiento académico: un estudio con universitarios. Revista Electrónica de la Federación española de Asociaciones de psicología, 10(7).

Delgado, A., Escurra, L., Bulnes, M., \& Quesada, R. (2001). Estudio psicométrico del Test de Matrices Progresivas de Raven forma avanzada en estudiantes universitarios. Revista de Investigación en Psicología, 4(2), 27-40.

Díaz, M., Valencia, G., Muñoz, J., Vivas, D., \& Urrea, C. (2006). Educación superior: horizontes y valoraciones, relación PEI-ECAES. Cali: Universidad de San Buenaventura, Facultad de Psicología Centro de Investigaciones Bonaventuriano e Instituto del Fomento de la Calidad de la Educación Superior, Icfes.

Díaz, D., Morales, M., \& Amador, O. (2009). Perfil vocacional y rendimiento escolar en universitarios. Revista Mexicana de Orientación Educativa, 6(16), 20-23.

Esparza, D., \& López, R. (2010). Perfil de ingreso de alumnos con buen desempeño académico en el primer año de estudios. El caso de la Escuela de Diseño de la Universidad De La Salle Bajío. Revista Electrónica Nova Scientia, 3(6), 95-120.

Ferreyra, M. (2007). Determinantes del Desempeño Universitario: Efectos Heterogéneos en un Modelo Censurado. (Tesis de Maestría en Economía, Universidad Nacional de La Plata). Recuperado de http://www.depeco.econo.unlp.edu.ar/maestria/ tesis/048-tesis-ferreyra.pdf

Garzón, R., Rojas, M., Del Riesgo, L., Pinzón, M., \& Salamanca, A. (2010). Factores que pueden influir en el rendimiento académico de estudiantes de Bioquímica que ingresan en el programa de Medicina 
de la Universidad del Rosario-Colombia. 2010. Revista Educación en medicina, 13(2), 85-96.

Hedel, R. (2003). El rendimiento académico: concepto, investigación y desarrollo académico. Revista electrónica sobre calidad, eficacia y cambio en educación, 1(2), 1-15

Hernández, J., \& Pozo, C. (1999). El fracaso académico en la Universidad: Diseño de un sistema de evaluación y detección temprana. Psicología Educativa, 5(1), 27-40. Recuperado de http://web.ebscohost. com/ehost/results?vid $=3 \&$ hid $=2 \&$ sid $=587899$ c 8 abb4-450b-b739-b8c4e88709e1\%40ses sionmgr107

Himmel citado en Reyes, Y. (2003). Relación entre el rendimiento académico, la ansiedad ante los exámenes, los rasgos de personalidad al autoconcepto y la asertividad en estudiantes de primer año de psicología de la UNMSM. (Tesis profesional para la obtención del grado de psicólogo, Universidad Nacional Mayor de San marcos, Perú).

Infante, M., Marín, M., \& Troyano, Y. (2000). El fracaso académico en la universidad: aspectos motivacionales e intereses profesionales. Revista Latinoamericana de psicología, 32(3), 505-517.

Instituto Colombiano para el Fomento de la Educación Superior ICFES. (2011). Examen de estado de la educación media. Resultados periodo 2005-2010. Bogotá, D.C.: ICFES. Recuperado de http://54.208.2.57/datos/Informe\%20resultados\%20historicos\%20Saber\%2011\%2020052010.pdf

OCDE y Banco Mundial. (2012). Evaluaciones de políticas nacionales de Educación. La Educación superior en Colombia. Bogotá: OCDE y el Banco Internacional de Reconstrucción y Fomento/el Banco Mundial.

Pereira, C., Hernández, G., \& Gómez, I. (2011). El valor predictivo de los exámenes de Estado frente al rendimiento académico universitario. Revista Educación y Educadores, 14(1), 51-65.

Piñero \& Rodríguez citados en Hedel, R. (2003). El rendimiento académico: concepto, investigación y desarrollo académico. Revista electrónica sobre calidad, eficacia y cambio en educación, 1(2). Recuperado de http://www.ice.deusto.es/RINACE/ reice/volln2/Edel.pdf

Raven J. C., Court, J., \& Raven, J. (1996). Raven Matrices Progresivas, Manual. Madrid: TEA Ediciones S.A.

Reyes, L. (2005). La deserción estudiantil en el programa de Psicología de la Corporación Educativa Mayor del Desarrollo Simón Bolivar, (Informe de autoevaluación). Barranquilla. Recuperado de http://www.alfaguia.org/alfaguia/ files/1320186525_26.pdf

Reyes, L., Castañeda, E., \& Pabón, D. (2012). Causas psicosociales de la deserción universitaria. Revista Logos Ciencia \& Tecnología. 4(1), 164-168. Recuperado de http://connection.ebscohost.com/c/ articles/87411278/causas-psicosociales-de-ladeserci-n-universitaria

Reyes, Y. (2003). Relación entre el rendimiento académico, la ansiedad ante los exámenes, los rasgos de personalidad al autoconcepto y la asertividad en estudiantes de primer año de psicología de la UNMSM. (Tesis profesional para la obtención del grado de psicólogo, Universidad Nacional Mayor de San marcos, Perú).

Roa, C., \& Vélez, A. (2005). Factores asociados al rendimiento en estudiantes de medicina de la Universidad del Rosario. Revista Educación Médica, $8(2), 74-82$

Roa \& Valero citados por V., J. (2006). Educación superior en el caribe colombiano: análisis de cobertura y calidad. Documentos de trabajo sobre economía regional, 69. Cartagena: Centro de Estudios Económicos - Banco de la República

Torres, L., \& Rodríguez, N. (2006). Rendimiento académico y contexto familiar en estudiantes universitarios. Revista Enseñanza e investigación en psicología, 11(2), 255-270.

Tursthone, L. (1989). Manual Inventario de Interés profesionales (IP). Madrid: Editorial TEA. 